\title{
Literature Overall of Fatigue Analysis Approaches of Vehicle Components Made of Rubber
}

\author{
V. TOMPOSNÉ SZÜLE \\ University of Széchenyi István, Faculty of Mechanical Engineering, Informatics and Electrical Engineering \\ Department of Applied Mechanics, szule.veronika@sze.hu
}

Abstract. Generally, the most frequently used structural materials are metals which have high strength and stiffness. However, there are many cases when other important properties come to the front, as well as high deformation capacity with elastic behavior, high viscosity namely good damping effect. Due to its above-mentioned properties, rubber is widely used in vehicle and automotive industry. Vehicle components made of rubber usually exhibit large deformations. Cyclic finite deformations generate temperature in hyperelastic materials. Furthermore it is necessary to take into consideration the effects of ambient temperature. The mechanical properties of rubber depend on temperature and temperature changes can accelerate chemical alteration processes which lead to the material deterioration and fatigue processes. Research on fatigue behavior and fatigue properties of rubber has a great significance for predicting fatigue life and improving durability of rubber products. There are several studies on the fatigue behavior of rubber-based materials, but there is less research in the fatigue life prediction considering the influence of temperature and temperature changes. First purpose of this paper is summarizing the influence of temperature and temperature changes on the fatigue behavior of rubber. The second purpose of this study is to provide an overview of the state of the art on the fatigue life prediction of rubber with primary focus on the different methods available for prediction of fatigue life under the influence of temperature and temperature changes.

\section{Introduction}

Fatigue life prediction at different temperatures has a profound significance for rubber parts to ensure their reliability and safety. Several studies can be found on the fatigue life of rubber but there is less research into fatigue life prediction considering temperature changes.

The fatigue life decreases when temperature increases. Thus, it is necessary to take into consideration the temperature changes during the fatigue process of rubber parts. In the case of rubber materials, different working temperatures will have different influences on the mechanical properties of rubber parts. Furthermore, another type of temperature changing has a significant role in the fatigue process of rubber. Cyclically loaded rubbers exhibit hysteretic response, showed by a stress difference between loaded and unloaded paths, and generated by the presence of the viscous stress in the rubber medium which deviates from the purely elastic response. In this case the temperature changing derives from the conversion of the dissipated mechanical energy (due to the hysteretic effect) into heat energy. The temperature changing may influence the rubber mechanical response due to its thermo-dependence [1]. 


\section{Fatigue analysis of rubber}

Two main approaches can be distinguished for analysing fatigue life in rubber components. These are the crack nucleation approach and crack growth approach. Elevated temperature has a deleterious effect on rubber, both on crack nucleation life, and on fatigue crack growth rate. The first approach considers an already existing crack with initial size and predicts the propagation of the crack until fracture (crack nucleation approach). The second approach focuses on predicting the growth of a particular crack (crack growth approach). The strength and limitations of these approaches are presented. Some information presented in this paper has been summarized previously $[4,5,6,7]$. Thus, this literature overall updates these above-mentioned reviews.

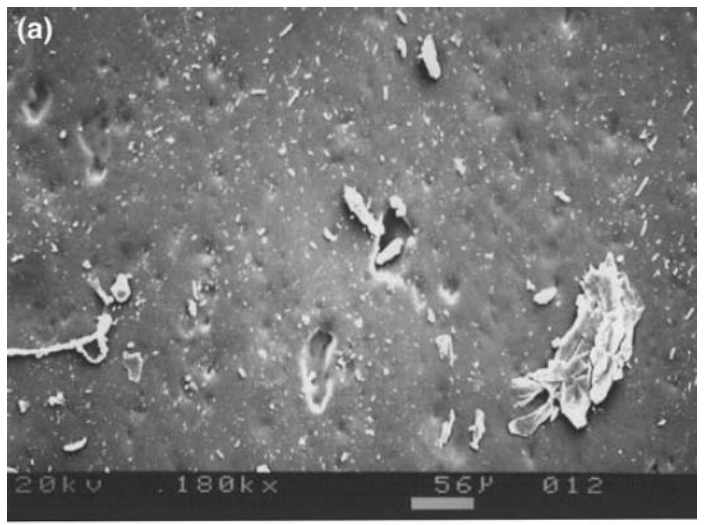

$\stackrel{0.056}{\longrightarrow} \mathrm{mm}$

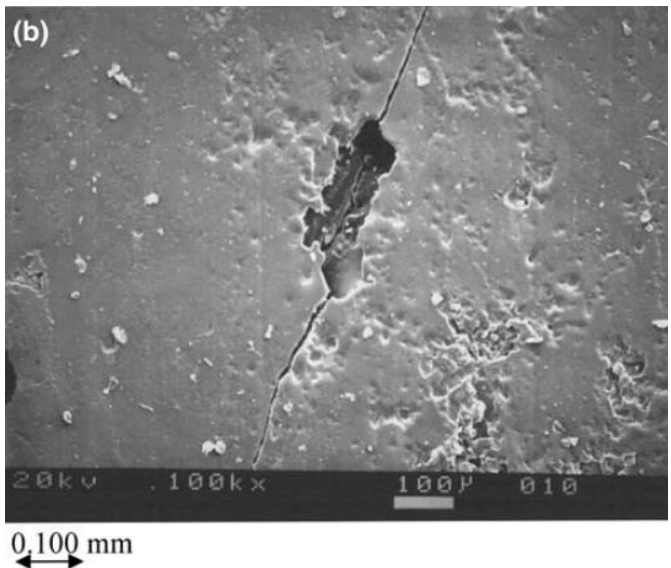

$\underset{\mathrm{0}}{0} 100 \mathrm{~mm}$

Table 1. Crack in rubber from voids [2,3]

\subsection{Crack nucleation theory}

According to the crack nucleation approach the considered material has an effective life which can be determined by the history of stresses or strains. The fatigue crack nucleation life can be determined by the number of cycles which cause the appearance of cracks with a critical size. The earliest academic study of fatigue was done by Wöhler in the 1860s, which was motivated by the understanding of fracture of railroad axles. An affine approach was applied to rubber in the 1940s [9]. There are two notable fatigue life parameters for predicting the fatigue life of rubber: the maximum principal strain and the strain energy density (SED) [8].

\subsubsection{Maximum principal strain}

Strain can be determined from displacements, which can be easily measured in rubber. Maximum principal strain is based on Cadwell's research in the 1940's on unfilled natural rubber. A good correlation was identified between the maximum principal strain and fatigue life in the case of tensile and shear loading of rubber. According to Cadwell, natural rubber has a superior fatigue endurance compared to synthetic rubber. During constant strain amplitudes the life of natural rubber improves as the minimum strain increases. Furthermore, in the case of rubbers that strain crystallize, increasing the minimum strain of the strain cycle can significantly elongate the fatigue life [10]. 


\subsubsection{Strain energy density}

Strain energy density proposes that the energy release rate is proportional to the product of SED and the crack size $[7,11]$. Several investigations can be found in literature which uses strain energy density as a fatigue life parameter in rubber. According to the Roberts and Benzies's studies in the case of NR and SBR (styrene butadiene rubber), the equibiaxial tension fatigue life is longer than simple tension fatigue life based on equal strain energy density. When the basis of the comparison is the maximum principal strain the result is the opposite. Roach investigated the cause of this above-mentioned difference and he found that all of the strain energy density is available for flaw growth in the case of simple tension, but only one half of the strain energy density is available in the case of equibiaxial tension.

It is important to note the critical plane approaches. The history of parameters associated with specific material planes are used to predict fatigue life in the case of multiaxial fatigue nucleation life of metals. However, in the case of rubber materials, multiaxial fatigue loading effects are not well understood. Compressive loading quite often occurs in the loading history of rubber parts. This type of loading along one direction is always related with simultaneous shear or tensile loading in other directions. Planes perpendicular to an axis of compression experience closure, planes in other directions experience shear or tension. Cracks will grow and nucleate on these planes.

\subsubsection{Cracking energy density}

The cracking energy density (CED) is part of the total elastic strain energy density, but concentrates on the energy available to a specific material plane during crack growth. CED is defined as the increase in energy density as the material is subjected to a fatigue load cycle:

$$
d W_{c}=t_{i} d \varepsilon_{i}, T=T_{c}
$$

where $t_{i}=\sigma_{i j} r_{j}$ is the traction stress on the plane with normal $r_{j}$ and $d \varepsilon_{i}=d \varepsilon_{i k} r_{k}$ is the change in traction strain on the plane. The energy in this plane is the portion of energy available to be released on a material plane.

To conclude, the classical crack propagation approach assumes a pre-existing crack, which grows until it reaches a failure size. This classical approach is difficult to use for rubbers, as the location of crack initiation could be hard to predict if only the principal strain and strain energy density is known. CED has been shown to be superior to the peak engineering strain as well as the energy density. CED gives superior results when fitting geometries with complex loading histories to a Wöhler curve, however CED is computationally expensive. It means iterating over a finite number of crack orientations in each element of a finite element model. The three fatigue evaluation criteria are compared in Table 1.

\begin{tabular}{|l|l|l|}
\hline Strain energy density & Maximum principal strain & Cracking energy density \\
\hline Depend on material & Depend on material and geometry & Less depend on material and geometry \\
\hline Worst correlation [12] & In simple load cases & Complex load cases \\
\hline Easy computing & Easy computing & $\begin{array}{l}\text { High computation times; } \\
\text { Implementation must be developed }\end{array}$ \\
\hline
\end{tabular}

Table 1. Comparison of fatigue life prediction parameters [12] 
Several applications of crack nucleation approaches are used in the rubber industry. However, the nucleation life approach to design analysis with rubber have not been very successful in the multiaxial loading cases of rubber components, based on the restricted data that have been reported for multiaxial conditions.

\subsection{Crack growth theory}

Several studies had gone into understanding the evolution of fatigue cracks in rubber materials. The crack growth theory considers pre-existing cracks or flaws.

According to Griffith's theory, the fracture criterion based on an energy balance including the mechanical energy of a cracked body and the energy associated with the crack surfaces. This approach was further developed for rubber by Thomas, Lake, Greensmith, Lindley, Mullins and Rivlin. Thomas extended this approach to analyse the growth of cracks under cyclic loadings in NR [12]. Thomas developed a square-law relationship between peak energy release rate and crack growth rate for unfilled natural rubber.

The cause of the growth of a crack microscopically is the crosslinks between the polymer chains in the rubber structure. These crosslinks are weak bonds and tear apart during subjected to high loads, as presented in Figure 1.

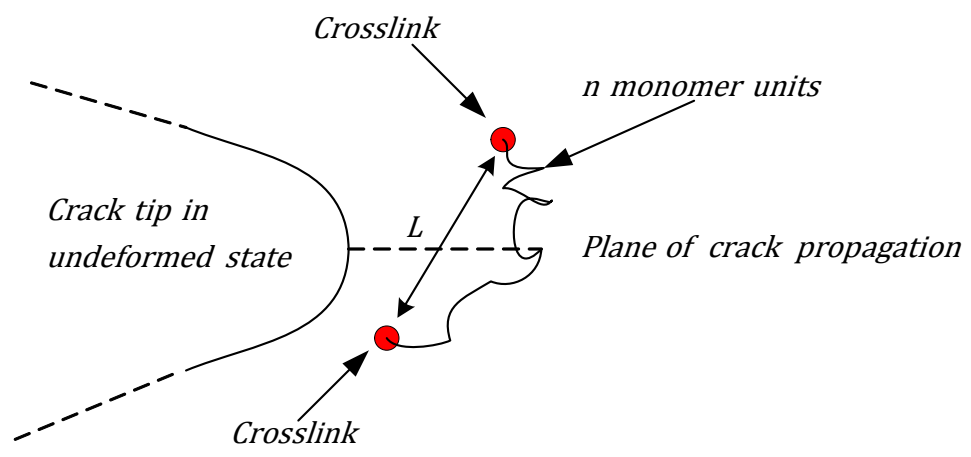

Figure 1. Crosslinks between the polymer chains when subjected to high loads. Adapted from [13]

The potential energy in rubber released from surrounding material is spent on reversible and irreversible changes to create new surfaces, according to Griffith's study. The crack growth is associated with new crack surfaces due to the conversion of a structure's stored potential energy. The growth of a fatigue crack is measured by the energy release rate, $T$. It means the measurement of the change in stored mechanical energy $d U$ per unit change in crack area $d A$.

$$
T=-\frac{d U}{d A}
$$

The energy is denoted $\mathrm{T}$, referring to the tearing energy required under static load. The crack growth rate is determined by the maximum energy release rate during pulsating loading [13]. Examples were studied by Greensmith and Lindley in which a single edge cut specimen with a crack of initial size $a_{0}$ subjected to tensile loading, as shown in Figure 2. 


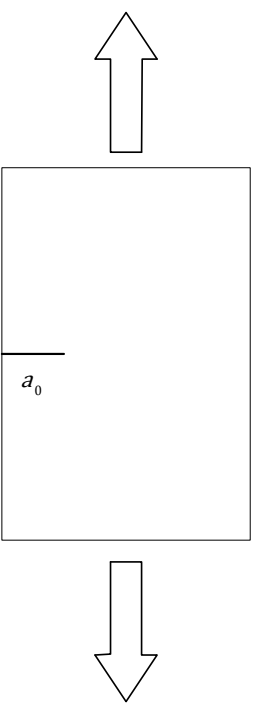

Figure 2. Plate with an initial crack $a_{0}$

The energy release rate was estimated as a function of the strain energy density, W, crack size $a$, and coefficient $k$, which is a function of the principal engineering strain.

$$
T=2 k W a
$$

\subsubsection{Regimes of fatigue crack growth}

Lake and Lindley distinguished four regimes of fatigue crack growth behaviour in rubbers as shown in Figure 3., based on the maximum energy release rate per cycle, $\mathrm{T}$, for $\mathrm{R}=0$ cycles, for unfilled NR and SBR [14].

- Regime 1: the crack growth rate $d a / d N$ below $T_{0}$ threshold is independent of the mechanical loading, and crack growth rate is independent of the energy release rate, $\mathrm{T}$. $\mathrm{T}$ is the rate at which energy per square unit is dissipated during fracture of the newly created fracture surface area. It means that rubber has no endurance limit. Furthermore, strain crystallization is a phenomenon that is specific to NR, and it is the primary cause of its unique superior fatigue resistance. Strain crystallization appears during compressive loading, which can have a retarding effect on crack growth rate by crystallization of the crack tip.

- Regime 2: is a transition range of $T$, between $T_{0}$ and $T_{t}$, described by the following relationship, in which $A$ is a material property.

$$
\frac{d a}{d N}=A\left(T-T_{0}\right)+r, T_{0} \leq T \leq T_{t}
$$

- Regime 3: is a range between $T_{t}$ and $T_{c}$ over which the relationship between the energy release rate and the fatigue crack growth rate suffice a power-law, where the material properties are $B$ and F.

$$
\frac{d a}{d N}=B T^{F}, T_{t} \leq T \leq T_{c}
$$

- $\quad$ Regime 4: beyond $T_{c}$ unstable crack growth regime follows, where the crack growth rate is infinite.

$$
\frac{d a}{d N}=\infty, T=T_{c}
$$


Several models have been developed to describe the last three regions with one relationship. The Crack-Layer theory for rubber is a single relationship that predicts regimes 2,3,4, for $R=0$ loading. This theory was developed by Aglan and Moet and is based on the irreversible thermodynamics of an "active zone" preceding the crack tip [15]. This is the most often used method of estimating life in practical applications, and there can be found many researches on obtaining power laws based of different geometries and load cases. There has not been found any unified method that accounts for the different fatigue effects of rubber. Fatigue correlations always depend on the geometry and composition of the specimen, the temperature, aging, and loading sequence order.

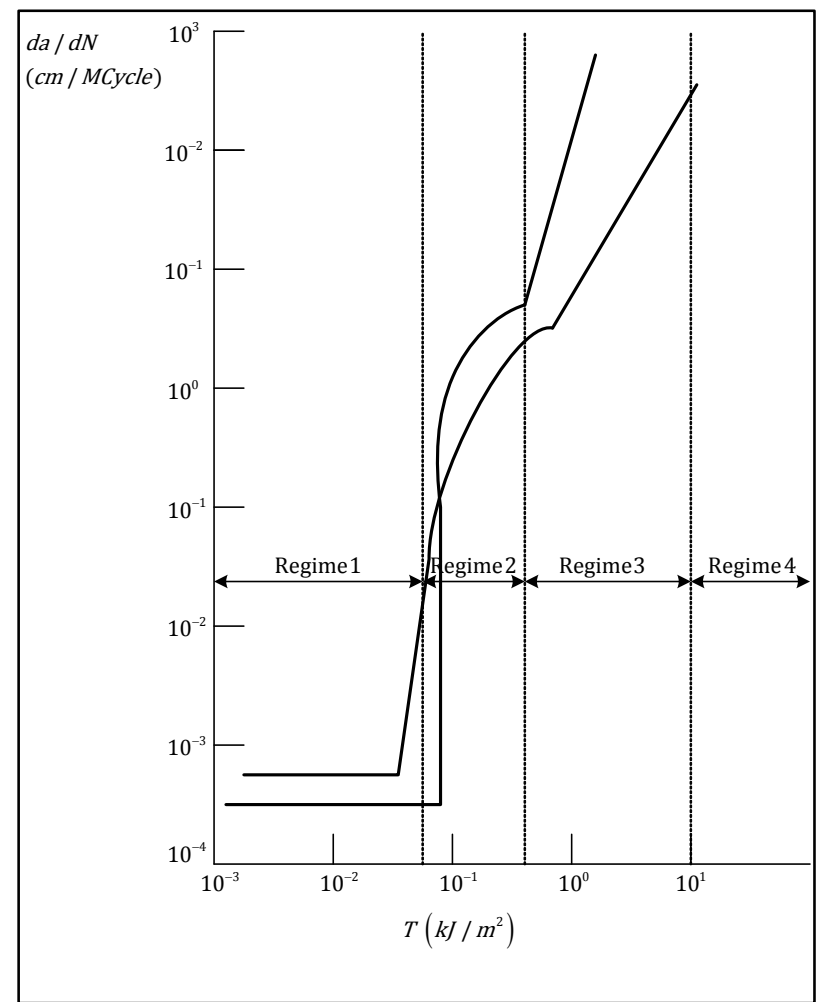

Figure 3. Four regimes of fatigue crack growth and their relation to the energy release rate. Adopted from [14]

\section{Effects of temperature}

Owing to their superior ability to good wear resistance and tear resistance, rubber materials are widely used in the automobile industry. For rubber materials, on the one hand, different working temperatures will have different influences on their mechanical properties. This is the first type of temperature changing. On the other hand, cyclically loaded rubbers exhibit hysteretic response, manifested by a stress between loading and unloading paths, and generated by the presence of the viscous stress in the rubber medium which deviates from the purely elastic response. A significant part of the dissipated mechanical energy due to the hysteretic effect may be converted into heat energy which manifests itself in the form of temperature changing. In the following section, the effect of temperature on the mechanical and fatigue behaviour of rubber will be investigated according to the state of the art.

- Temperature modifies the elastic response of rubber due to entropic effects and viscosity. 
- Temperature decreases and limits crystallinity. Treloar (1975) defined the temperature at which all the crystallites melt. According to Albouy et al. (2005) and Trabelsi (2002) SIC (strain-induced crystallization) almost completely disappeared.

Strain crystallization is an effect that is pertinent to fatigue in rubber. As the rubber stretched, some of the polymer chains in the amorphous material become aligned and produce crystalline structures oriented in the strain direction. These are called lamellae. The crystalline structure acts as a filler in the amorphous material and greatly increases the tensile strength. This is in turn increases the fatigue life. Strain crystallization occurs during high tensile loading and compressive loading, which can have a retarding effect on crack growth rate by crystallization of the crack tip.

- The crystallite melting temperature depends on the stretch, i.e. the higher the stretch, the higher the test temperature is needed to melt crystallites [16].

- Filled natural rubber can be used as good anti-vibratory part thanks to its property to resist crack propagation. These anti-vibratory parts undergo temperature up to $110^{\circ} \mathrm{C}$ in the engine environment, but only few studies occupied with the effect temperature on the lifetime reinforcement, although SIC (strain-induced crystallization) is a high thermo-sensitive phenomenon. Strong lifetime reinforcement was observed and attributed to SIC according to Fielding 1943 and Lindley 1974 [15, 16].

- Ruellan et al. investigated the temperature effects on the fatigue life reinforcement due to SIC for non-relaxing loadings. Strong lifetime reinforcement was obtained at $23^{\circ} \mathrm{C}$ during uniaxial fatigue tests. Fatigue damage mechanisms were investigated at the microscopic scale where the signature of reinforcement due to SIC (fatigue striations, cones) had been identified. Fatigue life reinforcement was still observed at $90^{\circ} \mathrm{C}$, and it was lower than $23{ }^{\circ} \mathrm{C}$, and fatigue life reinforcement totally disappeared at $110^{\circ} \mathrm{C}$ due to the disappearance of SIC at such temperature level [17].

Furthermore, it is necessary to take into consideration the effects of temperature under fatigue loadings:

- The fatigue resistance of NR decreases as the temperature increases (Lu, 1991).

- Le Chenadec (2008) determined the existence of a temperature threshold at $70^{\circ} \mathrm{C}$. The NR fatigue resistance is affected from this value.

- $\quad$ NR is highly subjected to self-heating. Lu (1991) and Xie (1992) has been measured a difference up to $50{ }^{\circ} \mathrm{C}$ between the bulk and the surface of a Diabolo. Thus, self-heating has to be taken into account in order to analyse the effect of temperature on the rubber fatigue behaviour.

- Fatigue tests carried out at elevated temperature generate thermal and thermo-oxidative aging. It means the failure and recombination of sulfur bonds, chain scission and additional vulcanization.

- When the working temperature increases, the thermal-oxidative aging reaction of rubber materials will accelerate. Zhang et al. carried out fatigue tests at elevated temperature which generated thermal and thermo-oxidative aging [18].

To conclude there are some areas in which future progress can be made, i.e.: in the area of SIC there are two unanswered questions: which are the conditions of fatigue-induced SIC and what is the minimum crystallinity level required to ensure reinforcement? 


\section{Prediction methods}

Fatigue life prediction due to temperature changes has a substantial significance for rubber components to ensure their reliability and safety. There are several studies on the fatigue life of rubber materials, but there is less research into fatigue life prediction considering temperature factors. Several scholars have performed their research on the prediction of rubber fatigue life.

- Zhang et al. established a fatigue life prediction model with strain energy as damage parameter and the fatigue characteristics under different temperatures are predicted by the relationship between their model parameters and temperature [17].

- Fatemi and co-workers determined a prediction method for rubber components in which the maximum principal strain was used as a damage criterion, and Miner's linear cumulative damage rule was applied based on the crack initiation life and fatigue crack growth [18].

- Woo et al. applied finite element analysis and life prediction of the rubber composites by using Green-Lagrange strain as fatigue damage parameter [19].

- Suryatal et al. predicted the fatigue life of a railway elastomeric pad by combining the experiment of material properties and using the Mooney-Rivlin model for FEA. Maximum first principal elastic strain was selected as the fatigue damage parameter [20].

- Seichter et al. summarized the advantages of fatigue crack growth theory. [21]

- Shangguan et al. predicted the fatigue life of a rubber isolator by choosing and analysing different fatigue damage parameters. [16]

- Wang et al. computed three fatigue damage parameters by finite element method. These are: logarithmic principal strain, Cauchy principal stress and strain energy density for fatigue life. This prediction model is based on the least-square method.

To conclude: the fatigue life forecast by the prediction models was compared with the experimental life, that showed that the prediction model which used the effective stress as damage parameter had the best accuracy [20].

At present, the prediction of rubber fatigue life under the influence of temperature is faced with two major difficulties. First of all, it is necessary to execute the suitable fatigue test and establish a model to analyse the fatigue characteristics of such parts because of the large variation of working temperature and complex loading conditions. Secondly, it is difficult to create the relationship between temperature and fatigue life model to predict the rubber fatigue life at different temperatures.

\subsection{Prediction methods under the influence of temperature}

Mars and Fatemi presented that the fatigue life of rubber material changes with temperature. Neuhaus et al. carried out fatigue tests to study the influence of chemical and thermal aging. Shangguan et al. established three kinds of fatigue life prediction models at different temperatures using the engineering strain as damage parameter [22].

These studies investigate the fatigue characteristics under the influence of temperature, but no model was established to demonstrate the relationship between temperature and fatigue life prediction. 
Zhang et al. established a fatigue life prediction model with strain energy as damage parameter and fatigue characteristics under different temperatures are predicted by the relationship between the model parameters and temperature.

Guo et al. investigated influence of temperature, viscoelasticity and damage on the rubber softening. They developed a new thermo-viscoelastic damage approach in accordance with thermodynamic principles for the prediction of fatigue-induced stress-soften. The proposed constitutive model is implemented into a finite element program. This new approach is able to predict the fatigue thermomechanical response rubbers. It means that cyclically loaded rubbers exhibit a historydependent response characterized by fatigue-induced stress-softening and hysteresis along with dissipative heating. These different inelastic phenomena are usually appearing together thus the coupling between them is justified. The fatigue induced stress softening is attributed to the network degradation and accumulation of damage effects within the rubber medium. The underlying physical process is attributed to both unrecoverable damage mechanisms and recoverable viscoelastic mechanisms. Cyclically loaded rubbers exhibit hysteretic response, manifested by a stress between loading and unloading paths, and generated by the presence of the viscous stress in the rubber medium which deviates from the purely elastic response. A significant part of the dissipated mechanical energy due to the hysteretic effect may be converted into heat energy which manifests itself in the form of temperature changing [23, 24].

Very few contributions dealing with the thermomechanical constitutive modelling of polymers. Most of these papers do not investigate the temperature changing due to dissipative heating. Guo et al. defined network thermal kinetics and network damage kinetics and used to alter the relaxed network during fatigue loading process. In order to take into consideration the rubber network properties, the relaxed free energy function is defined by Arruda and Boyce in this model. The average chain length and the average chain density are taken as functions of the temperature and fatigue damage $[25,26]$.

\section{Concluding remarks}

This paper summarized the influence of temperature and temperature changes on the fatigue behavior of rubber and provided an overview of the state of the art on the fatigue life prediction of rubber with primary focus on the different methods available for prediction of fatigue life under the influence of temperature and temperature changes. To conclude there are some areas in which future progress can be made, i.e.: in the area of SIC there are two unanswered questions: which are the conditions of fatigue-induced SIC and what is the minimum crystallinity level required to ensure reinforcement? Furthermore, the prediction of fatigue behaviour under complex mechanical loading histories has not been cleared, because multiaxial effects on crack nucleation life are not investigated. It is necessary to integrate environmental factors into fatigue life predictions. Models for the effects of temperature must be integrated with predictions on mechanical load history. Guo et al. developed a new approach which is able to predict the fatigue thermomechanical response in rubbers, and in which the authors used the Arruda-Boyce model to determine free energy functions. The question is raised what are the results of this approach using other models to predict the fatigue thermomechanical response $[17,26]$. 
This study is the basis of the following research which will include the modelling of the temperaturechanging induced fatigue behaviour and fatigue life prediction of rubberlike materials by using selfdeveloped finite element code.

\section{Acknowledgement}

This study is Supported BY the ÚNKP-19-3-III-SZE-7 New National Excellence Program of the Ministry For Innovation And Technology.

\section{References}

[1] X. Duan et al., The mechanical fatigue limit for rubber. Journal of Automotive Engineering, 7, 2015, 942-954.

[2] W.V. Mars, Chapter 7-Heuristic approach for approximating energy release rates of small cracks under finite strain, multiaxial loading. Elastomers and components (2006), 91-111.

[3] W. V. Mars and A. Fatemi, Nucleation and growth of small fatigue cracks in filled natural rubber under multiaxial loading. Journal of Materials Science 41 (22 2006), 7324-7332.

[4] Lake Gj., Fatigue and fracture of elastomers. Rubber Chemistry and Technology, (1995), 61, 503533.

[5] Lake Gj., Thomas Ag., Strength. In: Gent A, editor. Engineering with rubber, How to design rubber components. Munich: Carl Hanser Verlag, (1992), chapter 5.

[6] Lake Gj., Mechanical fatigue of rubber, Rubber Chemistry and Technology 1972, 45, 309-328.

[7] W.V.Mars, A.Fatemi, A literature survey on fatigue analysis approaches for rubber, International Journal of Fatigue 2002, 949-961.

[8] Wöhler A., Wöhler's experiments on the strength of metals. Engineering 1867, 2-160.

[9] Cadwell SM., Merrill RA., Sloman Cm., Yost Fl., Dynamic fatigue life of rubber. Industrial and Engineering Chemistry, Analytical Edition 1940, 12-23.

[10] Mars WV., Fatemi A., Factors that affect the fatigue life of rubber: literature survey. Rubber Chemistry and Technology, 2004, Vol. 77, 391-412.

[11] Gent An., Lindley Pb., Thomas Ag., Cut growth and fatigue of rubbers. I. The relationship between cut growth and fatigue. Journal of Applied Polymer Science, 1964, 455-466.

[12] Mars WV., Fatemi A., Multiaxial fatigue of rubber: Part I: equivalence criteria and theoretical aspects. Fatigue\&Fracture of Engineering Materials\&Structures 28, 2005, 515-522.

[13] Thomas Ag., Rupture of rubber. V. Cut growth in natural rubber vulcanizates., Journal of Polymer Science 1958, 31:467-480.

[14] Lake GJ., Lindley PB., The mechanical fatigue limit for rubber. Journal of Applied Polymer Science 1965, 9:1233-1251. 
[15] Aglan H., Moet A., The resistance of rubber compounds to brittle crack propagation. Rubber Chemistry and Technology 1989, 62:98-106.

[16] Ruellan B., J. B. Le Cam, Jeanneau I., Canévet F., Mortier F., Robin E., Fatigue of natural rubber under different temperatures, International Journal of Fatigue 2019, 544-577.

[17] Zhang J., Xue F., Zhang X., Han S., Strain energy-based rubber fatigue life prediction under the influence of temperature, Royal Society Open Science 2018, 1-13.

[18] Zarrin-Ghalami T., Fatemi A., Fatigue life predictions of rubber components: applications to an automobile cradle mount. Proc. Inst. Mech. Eng. D J. Automobile Eng. 2013, 691-703.

[19] Chang SW., Park Hs., Fatigue life evaluation of rubber-clay nanocomposites. Proc. Struct. Integr., 2016, 2173-2181.

[20] Suryatal B., Phakatkar H., Rajkumar K., Thavamani P., Fatigue life estimation of an elastomeric pad by $\varepsilon-N$ curve and FEA. J. Surf. Eng. Mater. Adv. Technol. 2015, 85-92.

[21] Seichter S., Archodoulaki VM., Koch T., Fidi W., Holzner A., Robin S., Experimental survey of approaches to estimate the uniaxial fatigue life of industrial rubbers. European Conference on Constitutive Models for Rubbers: Prague, Cz. 2015.

[22] Wang Wt, Xiao Sh., Huang Jl., Xie XX., Investigation on rubber isolator's fatigue life prediction under uniaxial tensile load, J. Vibr. Shock, 2015, 24-30.

[23] Wang W. Study on prediction of fatigue life of rubber mount based on linear cumulative fatigue damage theory, J. Mech. Eng, 2012, 56-65.

[24] Neuhaus C., Lion A., Johlitz M., Heuler P., Barkhoff M., Duisen F., Fatigue behaviour of an elastomer under consideration of ageing effects. International Journal of Fatigue, 2017, 104, 7280 .

[25] Shangguan WB., Li MM., Duan Xc., Fatigue life prediction and tests for rubber mounts under high temperature, J. Vibr. Shock, 2015, 66-71.

[26] Guo Q., Zairi F., Guo X., A thermo-viscoelastic-damage constitutive model for cyclically loaded rubbers. Part I: Model formulation and numerical examples, International Journal of Plasticity 2018, 106-124. 